\title{
Avaliação do perfil epidemiológico e da mortalidade dos pacientes com diagnóstico de melanoma cutâneo primário no município de Florianópolis - SC, Brasil
}

\author{
Evaluation of the epidemiological profile and the mortality rate of the patients \\ with primary cutaneous melanoma in Florianopolis - SC, Brazil
}

\author{
Rúbia Battisti ${ }^{1}$ \\ Ariana Lebsa Weber ${ }^{3}$ \\ Isadora Sgrott ${ }^{5}$
}

\author{
Daniel Holthausen Nunes ${ }^{2}$ \\ Louise Cardoso Schweitzer ${ }^{4}$
}

Resumo: Fundamentos: O melanoma é o câncer cutâneo com maior letalidade. Santa Catarina é o estado brasileiro com maior número de casos desse tumor.

OвJETIVOs: Estimar a taxa de mortalidade por melanoma no quinto ano de doença.

MÉTodos: A amostra compreendeu 81 laudos de melanoma primário cutâneo, em 75 pacientes, emitidos em Florianópolis - SC em 2002 e 2003. O protocolo de pesquisa incluiu idade, sexo, cor do paciente e localização anatômica, tipo histológico, grau de invasão, índice de Breslow, infiltrado inflamatório, ulceração, regressão, invasão angiolinfática e estadiamento do tumor. Foi feito contato telefônico com os pacientes para verificar seu status (vivo, morto por melanoma ou morto por outra causa). Para análise estatística, utilizou-se o teste exato de Fisher e a curva de sobrevida de Kaplan-Meier.

RESUltados: O perfil dos pacientes foi: feminino, branco, 51,3 anos, melanoma invasivo em tronco ou membros, tipo extensivo superficial, Breslow $2,63 \mathrm{~mm}$. A taxa de mortalidade por melanoma cutâneo foi de 7,0\%, maior entre homens (11,1\%), com Breslow superior a 4,0 $\mathrm{mm}(66,0 \%)$, com ulceração (33,3\%) e em estádio IV (80,0\%). A sobrevida média foi de 56,7 meses. Conclusões: A taxa de mortalidade por melanoma primário cutâneo foi de 7,0\%, e a ulceração e o estadiamento final foram os fatores com significância estatística sobre o resultado.

Palavras-chave: Análise de sobrevida; Coeficiente de mortalidade; Melanoma

\begin{abstract}
BACKGROUND: Melanoma is the cutaneous cancer which has the greatest lethality. Santa Catarina is the Brazilian State that contributes the most to increase this rate. Objectives: To estimate the mortality rate of melanoma in the fifth year of illness. Methods: The sample comprises 81 reports of primary cutaneous melanoma, in 75 patients, diagnosed in Florianopolis - SC in 2002 and 2003. The protocol of research includes age, sex, patient's color, anatomical location, histological type, degree of invasion, Breslow index, inflammatory reaction, ulceration, regression, angiolymphatic invasion and tumor staging. The patients were contacted by telephone to verify their status (alive, killed by melanoma or dead for another reason). Fisher's exact test and Kaplan-Meier's survival curve were used for the statistical analysis. Results: The patients' profile was: female, white, $\mathbf{5 1 . 3}$ years of age, with invasive melanoma on the trunk and limbs, extensive spreading type, Breslow $2.63 \mathrm{~mm}$. The mortality rate was $7.0 \%$, greater among men (11.1\%), Breslow above $4.0 \mathrm{~mm}$ (66.0\%), with ulceration (33.3\%) and stage IV (80.0\%). The average survival period was 56.7 months. Conclusions: The mortality rate of primary cutaneous melanoma was $7.0 \%$; ulceration and the final staging were the factors with statistic significance on the result.

Keywords: Melanoma; Mortality rate; Survival analysis
\end{abstract}

Recebido em 14.10.2008.

Aprovado pelo Conselho Consultivo e aceito para publicação em 29.05.09.

* Trabalho realizado no Hospital Universitário Polydoro Ernani de São Thiago, da Universidade Federal de Santa Catarina (HU-UFSC) - Florianópolis (SC), e no Instituto de Diagnóstico Anátomo-Patológico (Idap) - Florianópolis (SC), Brasil.

Conflito de interesse: Nenhum / Conflict of interest: None

Suporte financeiro / Financial funding: None

Aluna do sexto ano de graduação em Medicina da Universidade Federal de Santa Catarina (UFSC) - Florianópolis (SC), Brasil

Mestre e professor do Departamento de Clínica Médica, disciplina de Dermatologia da Universidade Federal de Santa Catarina (UFSC). Preceptor da Residência Médica de Dermatologia da UFSC. Professor de Dermatologia e Alergia Clínica da Universidade do Sul de Santa Catarina (Unisul) - Florianópolis (SC), Brasil. Médica residente de Infectologia do Hospital Nereu Ramos - Florianópolis (SC), Brasil.

Aluna do sexto ano de graduação em Medicina da Universidade Federal de Santa Catarina (UFSC) - Florianópolis (SC), Brasil

Aluna do sexto ano de graduação em Medicina da Universidade Federal de Santa Catarina (UFSC) - Florianópolis (SC), Brasil 


\section{INTRODUÇÃO}

Nas últimas décadas, o melanoma tornou-se um assunto de grande interesse devido ao aumento importante na sua incidência e à sua elevada letalidade. Embora represente apenas $4 \%$ a $5 \%$ dos casos de câncer de pele, o melanoma provoca a maioria das mortes por malignidades cutâneas, pelo seu alto potencial de enviar metástases a órgãos distantes. ${ }^{1}$

No Brasil as informações sobre o melanoma são limitadas, em âmbito tanto nacional como regional. Segundo dados do Inca, ${ }^{1}$ para o ano de 2008 são esperados 2.950 casos novos de melanoma em homens e $2.970 \mathrm{em}$ mulheres. As maiores taxas estimadas são para a região Sul. Algumas das razões para a elevada prevalência de melanoma no sul brasileiro podem ser a composição e os hábitos de sua população: uma maioria de caucasianos expostos à radiação solar durante vários meses por ano, devido às condições climáticas e às opções de lazer.

Tendo em vista esse contexto, o presente estudo elege como escopos analisar as características epidemiológicas, estimar a mortalidade até o quinto ano de doença e avaliar a sobrevida dos pacientes com o diagnóstico de melanoma primário cutâneo no município de Florianópolis, Santa Catarina, Brasil. Justificase o estudo pela ausência de dados catarinenses sobre o seguimento dos pacientes com melanoma, apesar de esse estado contribuir com importante parcela dos casos nacionais.

\section{CASUÍSTICA E MÉTODOS}

Foram selecionados para o estudo os pacientes com diagnóstico de melanoma primário cutâneo confirmado por histopatologia nos laboratórios de anatomia-patológica do Hospital Universitário Polydoro Ernani de São Thiago (HU-UFSC) e do Instituto de Diagnóstico Anátomo-Patológico (Idap), situados no município de Florianópolis - SC, entre os dias $1^{\circ}$ de janeiro de 2002 e 31 de dezembro de 2003.

Foram incluídos os pacientes cujo sítio primário do tumor era cutâneo, independentemente do sexo, idade, raça, procedência ou profissão. Excluíram-se os pacientes com diagnóstico de melanoma ocular, melanoma mucoso e recidiva de melanoma cuja lesão primária antecedia o ano de 2002. Os pacientes não localizados durante a pesquisa foram também excluídos.

Para a coleta de dados foi elaborado um protocolo constituído por uma série ordenada de questões, de caráter aberto ou de múltipla escolha, com as seguintes variáveis: idade, sexo, cor, status (vivo, morto por melanoma ou morto por outra causa), localização anatômica da lesão, tipo histológico, grau de invasão, índice de Breslow, infiltrado inflamatório, ulceração, regressão, invasão angiolinfática e estadiamento do tumor.
Os dados foram obtidos por análise do prontuário dos pacientes nas instituições onde foi realizado seu acompanhamento. Informações complementares foram coletadas com o próprio paciente ou com seu representante legal, por meio de ligação telefônica, na qual se explicou que o paciente teria liberdade de participação no estudo, sendo garantido sigilo de dados. Os pacientes receberam, por carta, um Termo de Consentimento Livre e Esclarecido.

As análises estatísticas foram realizadas com o software Stata versão 9.0. Para testar associação entre os resultados utilizou-se o teste exato de Fisher, considerando-se o nível de significância estatística de 5\%. Para avaliar a sobrevida dos pacientes fez-se uso da curva de Kaplan-Meier.

O estudo foi submetido e aprovado pelo Comitê de Ética da Universidade Federal de Santa Catarina, conforme o protocolo de número 327/07.

\section{RESULTADOS}

Foram selecionados 133 laudos de pacientes com melanoma maligno, totalizando 126 pacientes. Daqueles, excluíram-se 52 laudos: 19 por apresentarem melanoma metastático, um por tratar-se de recidiva de tumor cujo primeiro diagnóstico foi anterior a 2002, três por apresentarem melanoma mucoso e 29 por não se localizarem os pacientes. A amostra final contabilizou 81 laudos de melanoma primário cutâneo, correspondendo a 75 pacientes.

Dos 75 pacientes, 42 (56\%) eram mulheres e 33 (44\%), homens.

A idade dos pacientes, na ocasião do diagnóstico, variou de 17 a 83 anos, com média de 51,3. Para o sexo feminino, a idade variou de 17 a 83 anos (média de 50,42); para o masculino, a variação foi de 27 a 82 anos (média de 52,55).

Cinquenta e dois pacientes tinham pele de cor branca; não se registrou a cor da pele em 23 pacientes.

A localização do tumor variou entre seis regiões anatômicas: face (21 laudos; 25,9\%), pescoço (quatro laudos; 4,9\%), tronco (26 laudos; 32,1\%), membro superior (15 laudos; 18,5\%), membro inferior (14 laudos; $17,3 \%$ ) e nádegas (um laudo; 1,2\%). Entre os homens, ocorreu maior prevalência de melanoma no tronco $(21,0 \%)$ e face $(12,3 \%)$; para as mulheres, houve predomínio nos membros superiores e inferiores $(25,9 \%)$, também seguidos da face $(13,7 \%)$ (Tabela 1$)$.

Nos 81 laudos analisados, foram diagnosticados 38 melanomas in situ (46,9\%) e 43 invasivos $(53,1 \%)$.

Dos 43 casos de melanoma invasivo, o subtipo histológico extensivo superficial foi o mais prevalente, com 23 representantes $(53,5 \%)$, seguido do nodular, com 15 casos $(34,9 \%)$, do lentigo maligno melanoma, com três casos $(7,0 \%)$ e do acrolentiginoso, com dois representantes $(4,6 \%)$. 
TABela 1: Distribuição dos laudos de melanoma cutâneo primário emitidos em dois laboratórios de anatomiapatológica de Florianópolis - SC, no período de $1^{\underline{0}}$ de janeiro de 2002 a 31 de dezembro de 2003

( $\mathrm{n}=81$ laudos), conforme a localização do tumor por sexo

\begin{tabular}{|c|c|c|c|c|}
\hline \multirow[t]{2}{*}{$\begin{array}{l}\text { Sexo } \\
\text { Localização }\end{array}$} & \multicolumn{2}{|c|}{ Masculino } & \multicolumn{2}{|c|}{ Feminino } \\
\hline & $\mathbf{N}$. & $\%$ & N. & $\%$ \\
\hline Face & 10 & 12,3 & 11 & 13,7 \\
\hline Pescoço & 1 & 1,2 & 3 & 3,7 \\
\hline Tronco & 17 & 21 & 9 & 11,1 \\
\hline MMSS & 6 & 7,4 & 9 & 11,1 \\
\hline MMII & 2 & 2,5 & 12 & 14,8 \\
\hline Nádega & - & - & 1 & 1,2 \\
\hline Total & 36 & 44,4 & 45 & 55,6 \\
\hline
\end{tabular}

Em relação à espessura dos tumores invasivos, em apenas dois laudos o índice de Breslow não foi apresentado. A espessura variou de $0,13 \mathrm{~mm}$ até 40,0 $\mathrm{mm}$, com média de $2,63 \mathrm{~mm}$ e mediana de $1,25 \mathrm{~mm}$. Dezesseis laudos $(37,0 \%)$ apresentaram espessura menor ou igual a $1,0 \mathrm{~mm} ; 17(39,0 \%)$, espessura entre $1,01 \mathrm{~mm}$ e $2,0 \mathrm{~mm}$; cinco $(12,0 \%)$, espessura entre $2,01 \mathrm{~mm}$ e $4,0 \mathrm{~mm}$; e três $(7,0 \%)$, espessura superior a 4,0 $\mathrm{mm}$ (Tabela 2).

A presença de ulceração foi descrita em 12 dos 81 laudos $(14,8 \%)$. Em cinco laudos $(6,2 \%)$ havia presença de regressão na lesão; em apenas um $(1,2 \%)$ a invasão angiolinfática estava presente; em 59 tumores $(72,8 \%)$ foi encontrado infiltrado inflamatório (Tabela 3).

Dos 43 laudos com tumores invasivos, 26 apresentavam mitoses. Destes, 11 laudos mostravam cinco ou mais mitoses por campo de grande aumento (CGA) (Tabela 4).

Com relação ao estadiamento do tumor, registraram-se 38 lesões em estádio 0 (melanoma in situ, 46,9\%), 24 lesões em IA ou IB (29,6\%), 13 tumores em IIA ou IIB ou IIC (16,0\%) e 6 tumores em estádio IIIA ou IIIB ou IIIC, ou ainda em estádio IV (7,4\%) (Tabela 3).

No que concerne à avaliação da mortalidade, 65 pacientes $(86,0 \%)$ estavam vivos no quinto ano após o diagnóstico e os outros 10 (14,0\%) haviam ido a óbito nesse período. Destes, a causa mortis não foi desencadeada pelo melanoma em cinco $(7,0 \%)$; nos outros cinco pacientes $(7,0 \%)$, a razão do óbito estava diretamente relacionada à presença do tumor. Esses cinco pacientes correspondem a 6 laudos, uma vez que em um paciente que faleceu por melanoma foram detectadas 2 lesões uma in situ e outra invasiva. Para fins da análise de mortalidade, foram consideradas as características do tumor invasivo do paciente com as duas lesões.

A mortalidade por melanoma entre os homens foi de $11,1 \%$ e entre as mulheres, de $4,4 \%$.
Todos os cinco pacientes que faleceram por melanoma fazem parte do grupo de pacientes cujos tumores apresentavam infiltrado inflamatório; não houve óbitos por essa causa entre os pacientes sem infiltrado inflamatório (p: 0,182). Com relação à ulceração, quatro dos cinco pacientes que faleceram por melanoma apresentavam tumores ulcerados; apenas um paciente que foi a óbito por essa causa não apresentava o fator (p: 0,004). A regressão estava presente no laudo de apenas um paciente que foi a óbito por melanoma (p: 0,326). Também um único paciente cujo laudo demonstrava invasão angiolinfática faleceu por melanoma; outros quatro pacientes sem invasão à histopatologia morreram por essa causa (p: 0,074). (Tabela 3).

A mortalidade por melanoma entre os pacientes com tumores invasivos, no que se refere à presença de mitose, foi de um paciente sem mitoses $(5,9 \%)$ e quatro pacientes com mitose, sendo dois deles com menos de cinco mitoses/CGA $(13,3 \%)$ e os outros dois com cinco ou mais mitoses/CGA (18,2\%) (Tabela 4).

A mortalidade por melanoma em pacientes com lesões invasivas, no que concerne ao índice de Breslow, foi de um paciente $(6,3 \%)$ no grupo de espessura menor ou igual a $1,0 \mathrm{~mm}$, dois pacientes $(12,0 \%)$ no grupo de $1,01 \mathrm{~mm}$ a $2,0 \mathrm{~mm}$ e dois $(66,6 \%)$ entre aqueles com Breslow superior a 4,0 mm (Tabela 5).

Ao se avaliar a mortalidade de acordo com o estadiamento, observa-se que um paciente $(10,0 \%)$ em estádio IB e quatro $(80,0 \%)$ em estádio IV faleceram por melanoma (p: 0,001). Ressalta-se que um paciente desse último grupo apresentou ainda um segundo tumor, este em estádio 0 (in situ) (Tabela 3).

Em relação à sobrevida e considerando-se o acompanhamento dos pacientes com melanoma cutâneo primário desde a data da realização do laudo his- 
TABela 2: Distribuição dos laudos de melanoma cutâneo primário invasivo emitidos em dois laboratórios de anatomia-patológica de Florianópolis - SC no período de $1^{\circ}$ de janeiro de 2002 a 31 de dezembro de 2003 $(\mathrm{n}=43$ laudos $)$, conforme índice de Breslow

\begin{tabular}{|c|c|c|}
\hline \multirow{2}{*}{ Breslow* } & \multicolumn{2}{|c|}{ Número de Casos } \\
\hline & N. & $\%$ \\
\hline$\leq 1,0$ & 16 & 37 \\
\hline $1,01-2,0$ & 17 & 39 \\
\hline $2,01-4,0$ & 5 & 12 \\
\hline$\geq 4,01$ & 3 & 7 \\
\hline Sem registro & 2 & 5 \\
\hline Total & 43 & 100 \\
\hline
\end{tabular}

*Unidade: milímetros (mm)

topatológico até a ocasião do contato com esses pacientes, a sobrevida média foi de 56,74 meses. O gráfico 1 ilustra esses valores.

\section{DISCUSSÃO}

O presente estudo compreende uma amostra de 81 laudos histopatológicos de melanoma cutâneo, correspondendo a 75 pacientes. Nessa casuística, houve predomínio de lesões no sexo feminino, dado que concorda com os da literatura nacional. ${ }^{2-4}$

A média de idade foi de 51,3 anos, ratificando dados da literatura, que consagra adultos jovens como os mais susceptíveis para o melanoma. ${ }^{4-6}$ Embora esses resultados sejam classicamente conhecidos, ainda não se sabe ao certo o motivo pelo qual o melanoma mostra a tendência de aumento das taxas de diagnóstico na meia-idade. De fato seria de esperar uma maior ocorrência em faixas etárias mais avançadas, em virtude do efeito cumulativo da radiação solar e deterioração do sistema imune. ${ }^{7}$ Observou-se uma maior média de idade ao diagnóstico para o sexo masculino - 52,55 anos contra 50,42 anos para o sexo feminino -, porém essa diferença não é estatisticamente significante (p: 0,157).

Todos os pacientes questionados sobre a cor da pele eram brancos, dado também compatível com a literatura. ${ }^{8}$

Nesta casuística, maior percentual de pacientes com melanoma tinha como sítio primário da lesão o tronco, seguido pela face e membros. Entre as mulheres, houve predomínio de melanoma localizado em membros e face. No sexo masculino, os locais mais atingidos foram o tronco e a face (Tabela 1). Esses dados corroboram publicações atuais, nas quais os melanomas originam-se mais amiúde nos membros superiores e inferiores das mulheres e, com maior frequência, no tronco, cabeça e pescoço dos homens. ${ }^{9,10}$ As diferenças nos vestuários e nos estilos de vida são fatores que determinam as diferenças na topografia das lesões de acordo com o sexo. ${ }^{11}$

No que se refere à classificação histológica do tumor, mais da metade dos casos eram do subtipo extensivo superficial $(53,3 \%)$, seguido do nodular $(34,9 \%)$, do lentigo maligno melanoma $(7,0 \%)$ e do acrolentiginoso (4,6\%). O National Cancer Data Base Report, em revisão de 84.836 casos, apresentou dados semelhantes. ${ }^{12}$ Alguns estudos citam o melanoma nodular como o mais prevalente. ${ }^{13,14}$

Com relação ao grau de invasão, a maioria dos tumores apresentava invasão além da camada basal: $53,1 \%$ contra $46,9 \%$ dos casos de melanoma in situ. Sortino-Rachou et al. ${ }^{15}$ e Fernandes et $a l{ }^{3}$ encontraram proporção menor de tumores diagnosticados in situ, de apenas 2,8\% e 21,5\%, respectivamente. Esses dados, divergentes entre a literatura, podem ser decorrentes de particularidades de cada amostra, mas sugerem elevada suspeita dos médicos da cidade de Florianópolis para o diagnóstico de melanoma, confirmando-o de forma precoce.

Em 1970, Breslow ${ }^{16}$ descreveu um sistema de medição da espessura vertical do tumor em milímetros que permitia adequada reprodutibilidade entre os patologistas e que apresentava primorosa correlação com a sobrevida. Balch et $a l .{ }^{17}$ coletaram dados de 17.600 pacientes e ratificaram que a medida da espessura do tumor é o preditor mais forte do prognóstico dos pacientes com melanoma, por estar associada a um risco crescente de recorrência local e de metástases regionais e a distância. O prognóstico piora com a espessura crescente como uma função logarítmica contínua. Os pacientes da amostra foram divididos em quatro grupos, conforme a espessura de Breslow (Tabela 2). Dos 43 pacientes com melanoma invasivo, 41 tinham descrito esse índice em seus laudos. Destes, $76,0 \%$ apresentavam melanoma de pequena espessura ao diagnóstico $(37,0 \%$ menores ou iguais a $1,0 \mathrm{~mm}$ e $39,0 \%$ entre $1,01 \mathrm{~mm}$ e $2,0 \mathrm{~mm}$ ) e apenas $7,0 \%$ tinham tumores acima de $4,0 \mathrm{~mm}$. A média de Breslow encontrada foi de $2,63 \mathrm{~mm}$ com mediana de $1,25 \mathrm{~mm}$. Gon et al. ${ }^{9}$ e Criado et al..$^{10}$ encontraram porcentagens de lesões diagnosticadas com espessura 
TABela 3: Distribuição dos laudos de melanoma cutâneo primário emitidos em dois laboratórios de anatomiapatológica de Florianópolis - SC no período de $1^{\mathrm{o}}$ de janeiro de 2002 a 31 de dezembro de 2003 ( $\mathrm{n}=81$ laudos) segundo seu status no quinto ano do diagnóstico, conforme presença de infiltrado inflamatório, ulceração, regressão, invasão angiolinfática e estadiamento

\begin{tabular}{|c|c|c|c|c|c|c|c|c|}
\hline & \multicolumn{2}{|c|}{ Vivos } & \multicolumn{4}{|c|}{ Mortos } & \multicolumn{2}{|c|}{ TOTAL } \\
\hline & \multirow[b]{2}{*}{ N. } & \multirow[b]{2}{*}{$\%$} & \multicolumn{2}{|c|}{ por melanoma } & \multicolumn{2}{|c|}{ por outra causa } & \multirow[b]{2}{*}{$\mathbf{N}$. } & \multirow[b]{2}{*}{$\%$} \\
\hline & & & $\mathbf{N}$ & $\%$ & $\mathbf{N}$ & $\%$ & & \\
\hline \multicolumn{9}{|c|}{ I. inflamatório* } \\
\hline Presente & 50 & 84,8 & 6 & 10,2 & 3 & 5,1 & 59 & 100 \\
\hline Ausente & 20 & 90,9 & - & - & 2 & 9,1 & 22 & 100 \\
\hline \multicolumn{9}{|l|}{ Ulceração } \\
\hline Presente & 8 & 66,7 & 4 & 33,3 & - & - & 12 & 100 \\
\hline Ausente & 62 & 89,8 & 2 & 2,9 & 5 & 7,2 & 69 & 100 \\
\hline \multicolumn{9}{|l|}{ Regressão } \\
\hline Presente & 4 & 80 & 1 & 20 & - & - & 5 & 100 \\
\hline Ausente & 66 & 86,8 & 5 & 6,6 & 5 & 6,6 & 76 & 100 \\
\hline \multicolumn{9}{|c|}{ I. angiolinfática $\dagger$} \\
\hline Presente & - & - & 1 & 100 & - & - & 1 & 100 \\
\hline Ausente & 70 & 87,6 & 5 & 6,3 & 5 & 6,3 & 80 & 100 \\
\hline \multicolumn{9}{|l|}{ AJCC $\ddagger$} \\
\hline 0 & 34 & 89,5 & 1 & 10 & 3 & 7,9 & 38 & 100 \\
\hline IA & 13 & 92,9 & - & - & 1 & 7,1 & 14 & 100 \\
\hline IB & 9 & 90,0 & 1 & - & - & - & 10 & 100 \\
\hline IIA & 5 & 83,3 & - & - & 1 & 16,7 & 6 & 100 \\
\hline IIB & 6 & 100 & - & - & - & - & 6 & 100 \\
\hline IIC & 1 & 100 & - & - & - & - & 1 & 100 \\
\hline IIIB & 1 & 100 & - & - & - & - & 1 & 100 \\
\hline IV & 1 & 20 & 4 & 80 & - & - & 5 & 100 \\
\hline Total & 70 & 86,4 & 6 & 7,4 & 5 & 6,2 & 81 & 100 \\
\hline
\end{tabular}

* I. inflamatório: Infiltrado inflamatório

$\dagger$ I. angiolinfática: Invasão angiolinfática

$\ddagger$ AJCC: Estadiamento final segundo o AJCC, $2002^{25}$

menor do que $0,76 \mathrm{~mm}$ próximas às deste estudo (de $25,0 \%$ e $28,4 \%$ respectivamente) e da média nacional.

A proporção de mortes decorrentes do melanoma cutâneo em aproximadamente cinco anos foi de $7,0 \%$, com incidência de mortalidade da ordem de 0,014 óbitos por melanoma ao ano. Entre os indivíduos do sexo masculino, $11,1 \%$ faleceram devido ao tumor, contra apenas $4,4 \%$ de mortes por melanoma entre as mulheres. Diversos estudos atuais apresentam tendência de aumento da mortalidade por melanoma principalmente à custa desse índice entre os homens. ${ }^{18-20}$ Outras publicações, por sua vez, já apresentam dados de estabilização da mortalidade por melanoma, e até decréscimo quando analisado apenas o grupo do sexo feminino. ${ }^{21}$ Essa tendência decorre possivelmente da maior preocupação com a beleza e a saúde da pele entre as mulheres e geralmente coincide com incentivo às campanhas educacionais de prevenção do câncer de pele direcionadas ao público em geral.
A infiltração de linfócitos ao redor das células do melanoma está relacionada com um melhor prognóstico. ${ }^{22} \mathrm{~A}$ atual amostra evidenciou, entretanto, que todos os pacientes que faleceram por melanoma apresentavam infiltrado inflamatório na lesão (Tabela 3); porém, esse dado não foi estatisticamente significativo (p: 0,182). A ulceração, por sua vez, é uma característica que está associada a maior agressividade..$^{22,23}$ Nesta casuística, um terço dos pacientes $(33,3 \%)$ com ulceração faleceu por melanoma, número estatisticamente maior do que a proporção de óbitos entre os pacientes sem essa manifestação (p: 0,004; Tabela 3). Essas taxas corroboram os dados da literatura. Dos pacientes com regressão, apenas um morreu por melanoma; dentre aqueles sem regressão, foram quatro os óbitos pelo tumor (p: 0,326). O único paciente que apresentou invasão angiolinfática em seu laudo foi a óbito por melanoma, e, apesar de esse atributo ser considerado fator de mau prognóstico, essa associação não foi estatisticamente significativa (p: 0,074). 
TABELA 4: Distribuição dos laudos de melanoma primário cutâneo invasivo emitidos em dois laboratórios de anatomia-patológica de Florianópolis - SC no período de $1^{\circ}$ de janeiro de 2002 a 31 de dezembro de 2003

( $n=43$ laudos) segundo seu status no quinto ano do diagnóstico, conforme a presença de mitoses

\begin{tabular}{|c|c|c|c|c|c|c|c|c|}
\hline & \multicolumn{2}{|l|}{ Vivos } & \multicolumn{3}{|c|}{ Mortos } & & \multicolumn{2}{|c|}{ TOTAL } \\
\hline & \multirow[b]{2}{*}{ N. } & \multirow[b]{2}{*}{$\%$} & \multicolumn{2}{|c|}{ por melanoma } & \multicolumn{2}{|c|}{ por outra causa } & \multirow[b]{2}{*}{$\mathbf{N}$} & \multirow[b]{2}{*}{$\%$} \\
\hline & & & N. & $\%$ & $\mathbf{N}$. & $\%$ & & \\
\hline \multicolumn{9}{|l|}{ Mitoses/CGA* } \\
\hline Sem mitoses & 15 & 88,2 & 1 & 5,9 & 1 & 5,9 & 17 & 100 \\
\hline$<5$ & 12 & 80,0 & 2 & 13,3 & 1 & 6,7 & 15 & 100 \\
\hline$\geq 5$ & 9 & 81,8 & 2 & 18,2 & - & - & 11 & 100 \\
\hline Total & 70 & 86,4 & 6 & 7,4 & 5 & 6,2 & 81 & 100 \\
\hline
\end{tabular}

* /CGA: por campo de grande aumento

A mortalidade por melanoma foi equivalente entre os pacientes com menos de cinco mitoses/CGA e aqueles com cinco ou mais mitoses/CGA: dois casos para cada grupo (Tabela 4). O percentual no grupo dos pacientes com altas taxas mitóticas, no entanto, foi discretamente maior, corroborando estudos atuais que consideram elevado número de mitoses um fator de pior prognóstico em pacientes com melanoma.

A mortalidade foi crescente com o aumento da profundidade de Breslow. Embora em números absolutos as mortes entre pacientes com tumores de até $2,0 \mathrm{~mm}$ tenham sido maiores do que as mortes nos pacientes com lesões maiores do que $4,0 \mathrm{~mm}$ (três e dois, respectivamente), a proporção de mortes pelo número de representantes de cada grupo foi superior no último, demonstrando sua maior agressividade (Tabela 5). Garber et al..$^{24}$ e Balch et al. ${ }^{17}$ relatam que o risco de morrer por melanoma é linearmente crescente com o aumento da espessura do tumor, até atingir um platô, com aproximadamente 6,0 a $8,0 \mathrm{~mm}$.

Todos os pacientes foram estadiados conforme a versão final do AJCC. ${ }^{25}$ Apenas um paciente com melanoma em estádio 0 faleceu pelo tumor; no entanto, ele apresentou uma segunda lesão, com espessura de $40,0 \mathrm{~mm}$, presença de ulceração e em estádio IV, que, sem dúvidas, foi a responsável pelo óbito. Outro paciente com estadiamento inicial (IB) faleceu por melanoma $(10,0 \%)$. Os outros quatro óbitos $(80,0 \%)$ ocorreram em pacientes com tumores em estádio IV (Tabela 3). A análise estatística mostrou-se significante ao comparar a mortalidade por melanoma entre os diferentes estádios ( $\mathrm{p}$ : 0,001), revelando uma maior gravidade entre os pacientes com lesões avançadas. Balch et al.25 demonstrou que a mortalidade por melanoma em cinco anos variava desde valores de $4,7 \%$ a $11,0 \%$ para lesões IA e IB até valores de 81,2 a 90,5\% em tumores metastáticos (IV).
No que concerne à avaliação da sobrevida, sua frequência dentro de cinco anos de doença foi de $86,0 \%$, com média de 56,74 meses, valores que se aproximam aos encontrados em publicações mundiais. ${ }^{4,15,26}$ Entre as mulheres, a sobrevida foi de $88,9 \%$, superando a dos homens, $83,3 \%$. Os pacientes foram avaliados desde a data do laudo histopatológico até o momento em que saíram do estudo, ou por óbito ou pela ocasião do contato com o pesquisador, quando se considera a última data precisa em que o participante estava vivo. A pequena mortalidade dessa casuística fez a curva de Kaplan-Meier se manter em um patamar elevado (Gráfico 1). Pode-se observar que uma proporção próxima a 0,93 dos pacientes sobreviveu até o quinto ano após o diagnóstico. A

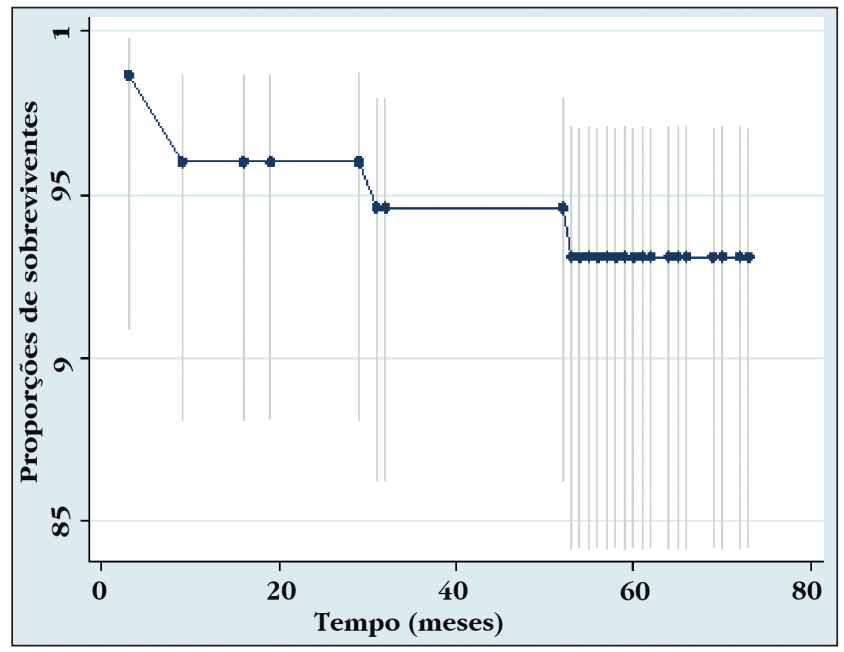

Gráfico 1: Proporção de sobreviventes conforme o tempo de acompanhamento entre pacientes com diagnóstico de melanoma cutâneo primário em dois laboratórios de anatomia-patológica de Florianópolis - SC no período de $1^{\circ}$ de janeiro de 2002 a 31 de dezembro de 2003 ( $n=75$ pacientes) 
TABela 5: Distribuição dos laudos de melanoma cutâneo primário invasivo emitidos em dois laboratórios de anatomia-patológica de Florianópolis - SC no período de $1^{\circ}$ de janeiro de 2002 a 31 de dezembro de 2003 ( $\mathrm{n}=43$ laudos) segundo seu status no quinto ano do diagnóstico, conforme o índice de Breslow

\begin{tabular}{|c|c|c|c|c|c|c|c|c|}
\hline & \multicolumn{2}{|c|}{ Vivos } & \multicolumn{4}{|c|}{ Mortos } & \multirow{2}{*}{\multicolumn{2}{|c|}{ TOTAL }} \\
\hline & \multirow[b]{2}{*}{ N. } & \multirow[b]{2}{*}{$\%$} & \multicolumn{2}{|c|}{ por melanoma } & \multicolumn{2}{|c|}{ por outra causa } & & \\
\hline & & & $\mathbf{N}$. & $\%$ & $\mathbf{N}$. & $\%$ & N. & $\%$ \\
\hline \multicolumn{9}{|l|}{ Breslow } \\
\hline$\leq 1,0$ & 14 & 87,4 & 1 & 6,3 & 1 & 6,3 & 16 & 100 \\
\hline $1,01-2,0$ & 15 & 88 & 2 & 12,0 & - & - & 17 & 100 \\
\hline $2,01-4,0$ & 4 & 80,0 & - & - & 1 & 20 & 5 & 100 \\
\hline$\geq 4,01$ & 1 & 33,4 & 2 & 66,6 & - & - & 3 & 100 \\
\hline Sem registro & 2 & 100 & - & - & - & - & 2 & 100 \\
\hline Total & 70 & 86,4 & 6 & 7,4 & 5 & 6,2 & 81 & 100 \\
\hline
\end{tabular}

relação entre variáveis pontuais do tumor e a evolução do paciente também se mostra bastante conveniente na confecção de curvas de sobrevida; o tamanho pequeno da amostra limitaria a precisão estatística do resultado e, portanto, este não foi apresentado.

A medida de maior efeito para diminuir as taxas de incidência e de mortalidade por melanoma e para aumentar a sobrevida livre de doença é a prevenção. A prevenção primária envolve evitar a exposição solar em horários de maior irradiação dos raios ultravioleta e estimular o uso de medidas físicas de proteção solar, como chapéus e sombrinhas, e também o uso de protetores solares. A prevenção secundária envolve diagnosticar precocemente lesões de pele malignas, com a participação dos profissionais de saúde e da própria população.

A exposição solar no Brasil é hábito cultural e está ligada a diversas atividades recreativas, e a proteção contra os efeitos deletérios do sol ainda é pouco valorizada. Essas características são marcantes no estado de Santa Catarina, ${ }^{27}$ tornando-o, junto com o Rio Grande do Sul, os estados brasileiros com maior prevalência de melanoma. ${ }^{1}$
É preciso desenvolver esforços junto à classe médica, aos responsáveis por tomadas de decisões no sistema de saúde e aos formuladores de políticas de educação para que a prevenção do câncer de pele seja incorporada ao cotidiano da população e iniciada já na infância, reduzindo, assim, a exposição cumulativa ao sol e suas consequências.

\section{CONCLUSÕES}

A mortalidade por melanoma primário cutâneo em pacientes diagnosticados no município de Florianópolis foi de 7,0\% no quinto ano de doença. A mortalidade por melanoma foi maior entre os homens, em pacientes com tumores de espessura superior a $4,0 \mathrm{~mm}(66,6 \%)$, com presença de ulceração $(33,3 \%$, p: 0,004$)$ e em pacientes com estadiamento IV $(80,0 \%$, p: 0,001).

A sobrevida média entre os pacientes com diagnóstico de melanoma primário cutâneo no município de Florianópolis foi de 56,74 meses. 


\section{REFERÊNCIAS}

1. INCA - Instituto Nacional do Câncer. [homepage] Câncer de pele melanoma. [Acesso em Set 2007]. Disponível em: http://www.inca.gov.br.

2. Lebsa-Weber A, Nunes DH, Souza Filho JJ, CarvalhoPinto CJ. Avaliação de 496 laudos anátomo-patológicos de melanoma diagnosticados no município de Florianópolis, Santa Catarina, Brasil. An Bras Dermatol. 2007;82:227-32.

3. Fernandes NC, Calmon R, Maceira JP, Cuzzi T, Silva CSC. Melanoma cutâneo: estudo prospectivo de 65 casos. An Bras Dermatol. 2005;80:25-34.

4. Borges SZ, Bakos L, Cartell A, Wagner M, Agostini A, Lersch E. Distribution of clinical-pathological types of cutaneous melanomas and mortality rate in the region of Passo Fundo, RS, Brazil. Int J Dermatol. 2007; 46:679-86.

5. Carvalho CA, Giugliani R, Ashton-Prolla P, Cunha ME, Bakos L. Melanoma hereditário: prevalência de fatores de risco em um grupo de pacientes no Sul do Brasil. An Bras Dermatol. 2004;79:53-60.

6. Bakos L, Wagner M, Bakos RM, Leite CS, Sperhacke CL, Dzekaniak KS, et al. Sunburn, sunscreens and phenotypes: some risk factors for cutaneous melanoma in southern Brazil. Int J Dermatol. 2002;41:557-62.

7. Dennis LK. Increasing risk of melanoma with increasing age. JAMA. 1999;282:1037-8.

8. Gloster HM Jr, Neal K. Skin cancer in skin of color. J Am Acad Dermatol. 2006;55:741-60.

9. Gon AS, Minelli L, Guembarovski AL. Melanoma cutâneo primário em Londrina. An Bras Dermatol. 2001;76:413-26.

10. Criado PR, Vasconcellos C, Sittart JAS, Valente NYS, Moura BP, Barbosa GL, et al. Melanoma maligno cutâneo primário: estudo retrospectivo de 1963 a 1997 no Hospital do Servidor Público Estadual de São Paulo. Rev Assoc Med Bras. 1999; 45:157-62.

11. Clark LN, Shin DB, Troxel AB, Khan S, Sober AJ, Ming ME. Association between the anatomic distribution of melanoma and sex. J Am Acad Dermatol. 2007;56:768-73.

12. Chang AE, Karnell LH, Menck HR. The National Cancer Data Base report on cutaneous and noncutaneous melanoma: a summary of $\mathbf{8 4 , 8 3 6}$ cases from the past decade. The American College of Surgeons Commission on Cancer and the American Cancer Society. Cancer. 1998;83:1664-78.

13. Pinheiro AMC, Cabral ALSV, Friedman H, Rodrigues HA. Melanoma cutâneo: características clínicas, epidemiológicas e histopatológicas no Hospital Universitário de Brasília entre janeiro de 1994 e abril de 1999. An Bras Dermatol. 2003;78:179-86.

14. Lapa MS, Guedes KF, Schalch FO, Landman G. Melanomas malignos cutâneos tratados no Hospital de Câncer de São Paulo. Estudo retrospectivo para avaliação, fatores prognósticos e sobrevida. An Bras Dermatol. 2002;77:313-20.

15. Sortino-Rachou AM, Curado MP, Latorre MRDO. Melanoma cutâneo: estudo de base populacional em Goiânia, Brasil, de 1988 a 2000. An Bras Dermatol.
2006;81:449-55.

16. Breslow A. Thickness, cross-sectional areas and depth of invasion in the prognosis of cutaneous melanoma. Ann Surg. 1970;172:902-8.

17. Balch CM, Soong SJ, Gershenwald JE, Thompson JF, Reintgen DS, Cascinelli N, et al. Prognostic factors analysis of 17,600 melanoma patients: validation of the American Joint Committee on Cancer melanoma staging system. J Clin Oncol 2001;19:3622-34.

18. Jemal A, Devesa SS, Fears TR, Hartge P. Cancer surveillance series: changing patterns of cutaneous malignant melanoma mortality rates among whites in the United States. J Natl Cancer Inst. 2000;92:811-8.

19. Hall HI, Miller DR, Rogers JD, Bewerse B. Update on the incidence and mortality from melanoma in the United States. J Am Acad Dermatol. 1999;40:35-42.

20. de Vries E, Schouten LJ, Visser O, Eggermont AM, Coebergh JW, Working Group of Regional Cancer Registries. Rising trends in the incidence of and mortality from cutaneous melanoma in the Netherlands: a Northwest to Southeast gradient? Eur J Cancer. 2003;39:1439-46.

21. Cohn-Cedermark G, Mansson-Brahme E, Rutqvist LE, Larsson O, Johansson H, Ringborg U. Trends in mortality from malignant melanoma in Sweden, 1970-1996. Cancer. 2000;89:348-55.

22. Ferreira CMM, Macieira JMP, Coelho JMCO. Análise imunohistopatológica, clínica e evolutiva dos melanomas. An Bras Dermatol. 1997,72:117-26.

23. Kufe DW, Ollock RE, Weichselbaum RR, Bast RC, Holland JF, Frei E, et al. Cancer medicine. 7th ed. Ontario, Canada: BC Becker; 2006.

24. Garbe C, Orfanos CE. Epidemiology of malignant melanoma in central Europe: risk factors and prognostic predictors. Results of the Central Malignant Melanoma Registry of the German Dermatological Society. Pigment Cell Res. 1992;Suppl 2:285-94.

25. Balch CM, Buzaid AC, Soong SJ, Atkins MB, Cascinelli $\mathrm{N}$, Coit DG, et al. Final version of the American Joint Committee on Cancer staging system for cutaneous melanoma. J Clin Oncol. 2001;19:3635-48.

26. Sim FH, Nelson TE, Pritchard DJ. Malignant melanoma: Mayo Clinic experience. Mayo Clin Proc. 1997;72:575-9.

27. Sociedade Brasileira de Dermatologia. Análise de dados das campanhas de prevenção de câncer de pele promovidas pela Sociedade Brasileira de Dermatologia de 1999 a 2005. An Bras Dermatol. 2006;81:533-9

ENDEREÇO PARA CORRESPONDÊNCIA / MAILING ADDRESS: Rúbia Battisti

Rua Marechal Deodoro, $n^{\circ}$ 555, Centro - Tijucas

88200000 Santa Catarina SC

Tel./Fax: 48 3263-1028 e $489907-7927$

E-mail:rubattisti@gmail.com

Como citar este artigo/How to cite this article: Battisti R, Nunes DH, Lebsa-Weber A, Schweitzer LC, Sgrott I. Avaliação do perfil epidemiológico e da mortalidade dos pacientes com diagnóstico de melanoma cutâneo primário no município de Florianópolis - SC, Brasil. An Bras Dermatol. 2009;84(4):335-42. 